\title{
BMJ Open Prevalence of latent TB infection and TB disease among adolescents in high TB burden countries in Africa: a systematic review protocol
}

Erick Wekesa Bunyasi, ${ }^{1}$ Bey-Marrie Schmidt, ${ }^{2}$ Leila Hussein Abdullahi, ${ }^{3}$ Humphrey Mulenga, ${ }^{1}$ Michele Tameris, ${ }^{1}$ Angelique Luabeya, ${ }^{1}$ Justin Shenje, ${ }^{1}$ Thomas Scriba, ${ }^{1}$ Hennie Geldenhuys, ${ }^{1}$ Robin Wood, ${ }^{4}$ Mark Hatherill ${ }^{1}$

To cite: Bunyasi EW, Schmidt B-M, Abdullahi LH, et al. Prevalence of latent TB infection and TB disease among adolescents in high TB burden countries in Africa: a systematic review protocol. BMJ Open 2017;7: e014609. doi:10.1136/ bmjopen-2016-014609

- Prepublication history and additional material is available. To view please visit the journal (http://dx.doi.org/ 10.1136/bmjopen-2016014609).

Received 11 October 2016 Revised 7 February 2017 Accepted 8 February 2017

CrossMark

For numbered affiliations see end of article.

Correspondence to Dr Mark Hatherill; mark.hatherill@uct.ac.za

\section{ABSTRACT}

Introduction: Almost a third of the world population has latent tuberculosis (TB) infection (LTBI), 10 million of whom develop TB disease annually, despite existence of effective, but lengthy, preventive and curative drug regimens. Although adolescents appear to have a very high force of LTBI, their reported incidence of TB disease is less than that of their corresponding general population. The few available studies on adolescent TB infection and disease prevalence are not sufficient to address the apparent discordance between rates of infection and disease in high TB burden countries in Africa. Therefore, we aim to perform a systematic review to examine the relationship between adolescent LTBI and TB disease, benchmarked against national TB disease burden data.

Methods and analysis: A comprehensive literature search will be performed for cross-sectional studies and screening data in cohort studies to determine the prevalence of LTBI and TB disease among adolescents in high TB burden countries in Africa in the following databases: PubMed, Scopus, Cochrane library, Web of Science, Africa Wide, CINAHL and the Africa Index Medicus. This will be supplemented by a search of reference lists of selected articles for potentially relevant articles. We will restrict our search to articles published in the English language between 1990 and 2016 among adolescents in order to obtain estimates reflective of the mature HIV epidemic in most high TB burden countries in Africa that occurred over this critical period. Primary end points are: prevalence of LTBI and TB disease. We will use the random-effects or fixed-effects modelling for our meta-analysis based on heterogeneity estimates.

Ethics and dissemination: No ethics approval is required given that this is a systematic review. Findings will be disseminated in a peer-reviewed journal in line with the Preferred Reporting Items for Systematic reviews and Meta-Analyses (PRISMA).

Trial registration number: CRD42015023495.
Strengths and limitations of this study

- To the best of our knowledge, this is the first systematic review to conduct and compare adolescent latent tuberculosis (TB) infection (LTBI) and $T B$ disease prevalence in high $T B$ burden countries in Africa.

- By examining the relationship between adolescent LTBI and TB disease benchmarked against national TB disease burden data, our study will provide key insights into this relationship.

- Data reporting adheres to the Preferred Reporting Items for Systematic reviews and Meta-Analyses (PRISMA) guidelines for reviews (PRISMA-R) and protocols (PRISMA-P).

- Our choice of period for review is primarily driven by the need to provide findings reflective of the mature HIV epidemic in high TB burden countries in Africa both before and after the advent of wide Anti-Retroviral Therapy (ART) availability; thus, we appreciate that our estimates will not provide old or historical trends in TB burden.

- Our restriction of analysis to articles published in the English language may introduce publication and language bias, respectively.

\section{INTRODUCTION}

Tuberculosis (TB) remains a key public health problem, especially in Africa, which reported almost a third of the 10.4 million incident TB disease cases globally in $2015 .{ }^{1}$ The estimated incidence rate of TB disease in Africa in 2015, of roughly 237 cases per 100000 people, was almost double the global average of 133 cases per 100000 people. ${ }^{1}$ In 2015, TB caused 1.4 million deaths worldwide and was the leading cause of death by an infectious agent. A relatively small proportion $(5-15 \%)$ of an estimated 2-3 billion people worldwide who are latently infected 
with Mycobacterium tuberculosis will develop TB disease in their lifetime. The probability of developing TB disease is much higher among people living with HIV. ${ }^{1}$

The force of TB infection, defined as the proportion of susceptible individuals (ie, individuals without latent TB infection (LTBI)) who become latently infected with M. tuberculosis per annum, is a key measure of TB transmission in a defined population. Unfortunately, very few longitudinal cohort studies of child or adolescent LTBI exist across high TB burden countries in Africa. A South African longitudinal study reported a high annual force of TB infection among adolescents of $14.0 \% .^{2}$ Similarly, there is a paucity of data on the prevalence of LTBI among adolescents in high TB burden countries in Africa, with most of the few available studies having been conducted in South Africa. A cross-sectional South African study reported an increase in prevalence of LTBI from $26 \%$ at 5-8 years to $53 \%$ at $14-17$ years to $75 \%$ at 25 years. ${ }^{3-7}$ A nationally representative Kenyan survey of children aged 6-14 years reported the prevalence of LTBI of $10.2 \%$, a figure that did not significantly change over two decades, between 1986 and 2006. ${ }^{8}$ Although adolescents in Cape Town, South Africa, appear to have a very high force of TB infection $(14 \%),{ }^{2}$ their reported incidence of TB disease $(\sim 710 /$ 100000 ) is less than the incidence in young adults $(1400 / 100000)$ and less than the incidence in the general population $(834 / 100000) .{ }^{12} 79$

A new TB infection in an infant or young child is a sentinel signal of active transmission from a person, usually an adult within their household, with active pulmonary TB disease. Thus, we would expect high rates of childhood LTBI to be associated with a high prevalence of adult TB disease in the same community. There is little research that describes settings from which adolescents acquire TB infection, ${ }^{10}$ which makes it difficult to explain the apparent discordance between very high rates of adolescent force of TB infection and low rates of notified adolescent TB disease in the same community. In a South African township, prevalent TB infection among children aged 5-14 years was directly and significantly associated with residential (ie, within their residential plot) exposure to an adult case of TB disease. However, a non-significant association was observed for individuals aged 15-22 years despite their high force of TB infection. ${ }^{10}$ This finding suggests the increasing significance of settings other than residential plot as a determinant of $\mathrm{TB}$ infection and subsequent disease from mid-adolescence onwards. ${ }^{11}{ }^{12}$ Glynn et $a l^{14}$ recently demonstrated via whole genome sequencing that, overall, known smear positive prior contacts accounted for $<10 \%$ of TB cases in a Malawian community, and that even for those with a prior contact with smear positive TB in their family, there was a higher than $50 \%$ chance that they acquired their TB elsewhere, similar to our own previous finding in Cape Town, South Africa. ${ }^{10} 13$ Andrews et at $t^{6}$ used statistical modelling techniques to estimate that up to a half of $\mathrm{TB}$ transmission among individuals aged 15-19 years occurs in the school setting, with this figure being $25 \%$ in individuals aged $0-14$ years. If this hypothesis were true, we would expect to observe a high prevalence of TB disease in parallel with a high force of TB infection among high school-aged adolescents in the same high burden communities. The fact that this apparently reasonable observation does not appear to hold true deserves further investigation. Our study will quantify the prevalence of LTBI and TB disease among adolescents in high TB burden countries in Africa and highlight this pattern across these countries. However, we appreciate that the design of this systematic review may not provide definitive reasons for this paradoxical yet persistent observation across many countries and settings. Owing to the lack of a systematic review on the prevalence of LTBI and TB disease among adolescents, this systematic review will provide useful data for policy by consolidating and synthesising available data regarding a key subpopulation with the highest force of TB infection ${ }^{2}$ but a relatively low reported notification rate of $\mathrm{TB}$ disease as compared with their corresponding general population. Our findings will contribute to our better understanding of TB transmission among adolescents, as well as inform TB policies in high TB burden countries in Africa by providing a reference for monitoring future TB transmission trends in the wake of global efforts to end the TB epidemic whose targets are defined in sustainable development goals for 2035. ${ }^{1}$ Our findings will also be useful in the planning of novel TB vaccine research studies among adolescents who are increasingly becoming a key focus subpopulation for global TB vaccine research efforts.

\section{METHODS AND ANALYSIS}

This protocol was developed in line with the Preferred Reporting Items for Systematic reviews and Meta-Analyses guidelines for protocols (PRISMA-P) ${ }^{15}{ }^{16}$ (see online supplementary file 1 for a PRISMA-P checklist of the recommended bare minimum items to be included).

\section{Objectives}

\section{Primary objectives}

- To determine the prevalence of LTBI in adolescents in the 25 high TB burden countries in Africa, as defined by the WHO in the 2016 Global TB report.

- To determine the prevalence of TB disease among adolescents in the 25 high TB burden countries in Africa, as defined by the WHO in the 2016 Global TB report.

\section{Secondary objective}

- To explore the relationship between age-specific risk of LTBI and age-specific prevalence of TB disease, benchmarked against published estimates of national TB disease incidence and notification rates. 


\section{Definitions}

Prevalence of LTBI is defined as the number of individuals with LTBI divided by the total number of individuals in a cross-sectional, population-based study or screening database in cohort studies with a positive or negative result from a diagnostic test for LTBI. We will consider LTBI diagnosed by the Tuberculin Skin Test (TST) and/or the Interferon Gamma Release Assay.

Prevalence of TB disease is defined as the number of individuals with $\mathrm{TB}$ disease divided by the total number of individuals in a cross-sectional, population-based study or screening database in cohort studies. We will consider the following diagnostic modalities for TB disease: solid and liquid mycobacterial culture, Xpert mycobacterium tuberculosis/rifampicin (MTB/RIF) assay, sputum smear for acid-fast bacilli and clinical diagnosis. Studies restricted to one or more forms of nonpulmonary TB disease only, for example, Koch's disease, TB lymphadenitis or disseminated TB, will not be included. Studies reporting on respiratory diseases in general and not clearly defining the prevalence of LTBI or TB disease will not be eligible.

Adolescents will be defined as individuals aged between 10 and 19 years, as defined by the WHO. ${ }^{17}$

In 2016, the WHO defined 'high TB burden countries' along three broad categories that included: (1) countries with the highest burden of TB/HIV coinfection, (2) countries with the highest burden of multidrug-resistant $\mathrm{TB}$ and (3) countries with the highest burden of TB. This classification takes consideration of the absolute number of cases of TB disease and the relative burden of $\mathrm{TB}$ disease after factoring the population size or denominator. In this study, we will restrict our review to the 25 countries from across these three WHO high TB disease burden categories that are found on the African continent. ${ }^{1}$ These include: (1) the Democratic Republic of Congo, (2) Ethiopia, (3) Kenya, (4) Uganda, (5) United Republic of Tanzania, (6) Zimbabwe, (7) South Africa, (8) Mozambique, (9) Angola, (10) Sierra Leone, (11) Central African Republic, (12) Congo, (13) Lesotho, (14) Liberia, (15) Namibia, (16) Zambia, (17) Botswana, (18) Cameroon, (19) Chad, (20) Ghana, (21) Guinea-Bissau, (22) Malawi, (23) Swaziland, (24) Somalia and (25) Nigeria.

\section{Criteria for consideration of studies for this review (eligibility criteria) \\ Study designs}

We will consider cross-sectional or prevalence study designs and screening data in cohort studies that report primary data on the prevalence of LTBI or TB disease. Statistical or mathematical modelling articles, costeffectiveness studies, opinion pieces, narrative reviews, case studies, case series and letters to editors will not be considered. Grey/unpublished literature will also be excluded.

\section{Participants}

Adolescent participants should be representative of the general adolescent population in the setting in which the study was conducted. Studies conducted among the general schoolgoing population will also be considered provided that age is reported. For studies that report on age ranges that extend beyond the age bracket from 10 to 19 years, data on individuals aged $10-19$ years will be extracted, if possible. Otherwise, these data will be sought from corresponding authors. If extraction is not possible and these data are not obtainable from the corresponding authors, at least $75 \%$ of participants should fall between the ages of 10 and 19 years. Studies reporting the prevalence of TB infection or TB disease in subpopulations that are not representative of the general adolescent or schoolgoing population in a specific study setting will be excluded, for example, studies reporting the prevalence of TB restricted to HIV-positive adolescents only.

\section{Outcome measures}

Outcome measures of interest will include prevalence of LTBI and TB disease. Studies which do not measure any of our primary outcomes; do not clearly state the case definition of LTBI or TB disease; do not report primary data; or lack explicit description of methodology, will be excluded.

\section{Time frame}

We will consider studies reported between 1 January 1990 and 1 July 2016 because this period will also reflect the TB burden in mature or generalised HIV epidemics across the high TB burden countries in Africa.

\section{Study setting}

Studies should have been performed in at least 1 of the 25 high TB burden countries in Africa as defined above. ${ }^{1}$ Studies not conducted in one of these countries or, for multicountry studies, if data pertaining to the listed high TB burden countries in Africa are not obtainable, they will be excluded.

\section{Language}

We will only consider articles published in the English language because of the limited time and financial resources available to this study.

\section{Search strategy}

We will systematically search for articles published between 1990 and 2016 using a combination of database-specific medical subject headings (MeSH terms) and a range of free-text or key words that will include the following, among others: adolescents, persons, latent, TB, LTBI, epidemiology, prevalence, morbidity and burden. Our draft PubMed search term is provided in online supplementary file 2 . The specific search strategies will be finalised with guidance from a health sciences librarian with expertise in systematic 
review searching with input from the project team. After the PubMed strategy is finalised, it will be adapted to the syntax and subject headings of the other targeted databases. We will review reference lists of selected articles to identify potentially relevant articles to our research questions that would have been missed by our search term in specified bibliographic databases. Our search will be limited to the following electronic databases due to the limited time and financial resources: PubMed, Scopus, Web of Science, Cochrane library, Africa Wide, Africa Index Medicus and CINAHL. This review will not include grey/ unpublished reports due to the low likelihood of peer review and potential practical difficulties of obtaining supplementary or missing data. We appreciate that this may lead to publication bias and acknowledge this as a limitation of our planned review.

\section{Selection of studies}

The first author (EWB) will perform a systematic search for articles by employing the search strategy. For duplicate articles or publications reporting the same data in multiple articles, only the recent and/or complete version of the publication will be considered. EWB will review references of selected articles to identify articles relevant to our review which would have been missed by the search strategy. EWB and B-MS will independently classify articles as: (1) 'included', (2) 'excluded' or (3) 'pending'. A 'pending' status shall imply that the reviewer is unsure on whether to include or exclude an article. This classification will be done by applying the inclusion and exclusion criteria, and will initially be based on the title and abstract, and then a quick scan, assessment or reading of the full text of the articles. Articles that both reviewers classify as 'excluded' will be excluded from further consideration, whereas those that both reviewers classify as 'included' will be included in the review. We will obtain full reports for all 'included' titles and those with contradictories in classification between the two reviewers. We will seek additional information from study authors where necessary to resolve questions about eligibility. A discussion will be held between EWB and B-MS to resolve differences or contradictories in classification of articles by reviewing the full text. A third reviewer (LHA) will be consulted to resolve persistent disagreements following discussion. We will present a flow chart, in keeping with Preferred Reporting Items for Systematic reviews and MetaAnalyses (PRISMA) guidelines as much as practicable, to summarise the search process and selection of studies for the review and document reasons for exclusion of studies (see online supplementary file 3 ). We will include a table of all selected studies in the final review and document reasons for exclusion of articles.

\section{Data management}

Data management will be done by the first author (EWB) in liaison with the second author (B-MS). A Google drive electronic folder will be maintained for the review and will contain: the protocol, a record of obtained articles and documentation of steps in data synthesis and analysis (including records included and excluded), risk of bias and quality scoring, among others. A back-up of the electronic records will be stored on a laptop and on a memory flash drive. 'Refworks' bibliographic management software ${ }^{18}$ will be used to manage references.

\section{Data extraction}

EWB will read, extract and collate data from selected articles on to a standardised data extraction form (see online supplementary file 4 ). This form will be piloted on at least four randomly selected studies meeting the criteria for consideration. B-MS will verify abstracted data in order to reduce bias and errors in data extraction. Data to be abstracted will include: study characteristics-title, year of publication, authors, study design; study setting and population-country, sociodemographics (age and gender); study conduct-number of study participants (total in the study and those participants with TB, by diagnostic approach and number with LTBI). Reviewers will resolve disagreements by discussion, with arbitration by LHA for unresolved disagreements. We will contact study authors for data that may resolve any uncertainties.

\section{Approach to missing data}

In the event of missing data that are key, we will attempt to contact the corresponding authors of the studies to obtain the relevant missing data via email. A second email will be sent after 1 week of the first email in the event of no response to the first email. A 2-week wait period from the date of submission of the second email will be allowed for responses, failing which these studies will be excluded, if no communication or response is established.

\section{Assessment of risk of bias of included studies}

Risk of bias and assessment of quality will be evaluated using an assessment tool adapted from Hoy et $a l^{19}$ by Werfalli $e t a l^{20}$ who included a scoring system for evaluation of prevalence studies. The tool helps evaluate internal and external validity (see table 1 ). This tool was preferred over others because it was designed via an expert consensus exercise and then tested, retested, validated and thus optimised for evaluation of quality of prevalence studies via a rigorous published process that included a review of limitations of existing tools. ${ }^{19} 21$ The tool was shown to have a high inter-rater agreement. ${ }^{19}$ Two authors (EWB and B-MS) will independently score the risk of bias using this tool and the mean score calculated. Agreement between the two raters will be assessed for each item in the tool and overall using the proportion of agreement $\left(\mathrm{P}_{0}\right)$ and the $\kappa$ statistic. For the $\kappa$ statistic, its values range from -1 to +1 . Values of 0 or less will be regarded as poor agreement, 0.01-0.20 slight, $0.21-0.40$ fair, $0.41-0.60$ moderate, $0.61-0.80$ 
Table 1 Risk of bias and quality assessment criteria for prevalence studies

Item under review

Quality score

(points)

External validity

Was the study's target population a close representation of the national population in relation to

1 relevant variables?

Was the sampling frame a true or close representation of the target population?

Was some form of random selection used to select the sample, OR was a census undertaken?

Was the likelihood of non-response bias minimal?

Total

Internal validity

Were data collected directly from the participants (as opposed to a proxy)?

Was an acceptable case definition used in the study?

Was the study instrument that measured the parameter of interest shown to have validity and reliability?

Was the same mode of data collection used for all subjects?

Was the length of the shortest prevalence period for the parameter of interest appropriate?

Were the numerator(s) and denominator(s) for the parameter of interest appropriate?

Total

Summary item on the overall risk of study bias (low, moderate or high)

As described by Hoy et al, the summary assessment evaluates the overall risk of study bias and is based on the rater's subjective judgement given responses to the preceding 10 items. This approach is consistent with the Cochrane and GRADE working group ${ }^{24}$ recommendation or approaches. Furthermore, as summarised in the PRISMA elaboration document, summative scales that numerically summarise multiple components into a single number are misleading and unhelpful, ${ }^{25}$ hence our choice of an overall ordinal scale for risk of bias. Response options for individual items are either low (1) or high risk of bias (0). If there is insufficient information in the article to permit judgement of a particular item, then the article is deemed to be at high risk of bias with respect to that item. ${ }^{19} 2627$

GRADE, Grading of Recommendation, Assessment, Development and Evaluation; PRISMA, The Preferred Reporting Items for Systematic reviews and Meta-Analyses.

substantial and $0.81-0.99$ almost perfect agreement. ${ }^{22}$ Raw agreement and $\kappa$ values (including their $95 \%$ CIs) will be calculated using STATA V.14.0 for Windows. ${ }^{23}$ Neither of the review authors will be blinded to the journal titles or to the study authors or institutions.

\section{Data analysis}

We hypothesise that there will be substantial statistical heterogeneity in study results because the prevalence of LTBI and TB disease varies by distribution of socioeconomic determinants of health and HIV prevalence within and across settings, among other factors. A priori, random-effects meta-analysis will be preferred due to the anticipated heterogeneity. However, choice of randomeffects or fixed-effects modelling will be based on observed statistical heterogeneity. For the latter, we will not pool the results but summarise findings in a narrative format. Additionally, we will derive annual risk of LTBI using the formulae: $1-(1-\text { prevalence })^{1 /(\text { mean age })}$ for every year of adolescence. We will then describe the relationship between the annual risk of TB infection and observed TB prevalence from our review. Alternatively, for countries with insufficient data, we will describe the relationship between the annual risk of TB infection and reported TB notification (or incidence rates estimates) by national TB programmes or estimates from the WHO.

In random-effects modelling, effect measures are assumed to vary between studies and the summary effect is the weighted average of the effects reported in different studies. ${ }^{28}$ This model directly adjusts for the inverse of the SE, and thus indirectly for the sample size reported in studies. Thus, studies with smaller SE and larger sample sizes will be given more weight in the calculation of the pooled prevalence and 95\% CIs.

\section{Data synthesis}

Our outcome will be combined and calculated using the Cochrane Review Manager (RevMan) statistical software, ${ }^{29}$ according to the statistical guidelines in the Cochrane Handbook for Systematic Reviews of Interventions. ${ }^{26}$ If statistical heterogeneity is observed, the randomeffects model will be chosen over the fixed-effects model. If there is substantial statistical heterogeneity, we will not perform a meta-analysis; a narrative, qualitative summary will be done supported by a table (see online supplementary file 5) and figures, where appropriate. This will be done by the first reviewer and checked by the second reviewer for accuracy.

\section{Assessment of reporting biases}

The potential for publication or reporting bias will be explored by funnel plots if we obtain at least 10 articles. This will be done by visually assessing asymmetry of funnel plots. As suggested by Egger et al, ${ }^{30}$ asymmetry of funnel plots will indicate the presence of publication bias. We appreciate that our choice of considering articles reported in English only (language bias) and the fact that we are only searching in a sample of bibliographic databases may be a source of reporting bias. 


\section{Assessment and management of heterogeneity}

We anticipate clinical and statistical heterogeneity in prevalence rate estimates within and across settings and countries. Statistical heterogeneity will be quantified using the $\mathrm{I}^{2}$ test statistic to determine the extent of variation in effect estimates that is due to heterogeneity rather than chance. Statistical heterogeneity will be explored graphically by inspection of forest plots (ie, the eyeball test). Non-overlap of 95\% CIs will suggest remarkable heterogeneity. A formal test for statistical homogeneity, the Cochran's $\chi^{2} \mathrm{Q}$ test statistic, will be performed using an $\alpha$ cut-off level of $10 \%$ as suggested by Higgins $e t a l^{1}$ and the Cochrane handbook, ${ }^{32}$ due to the test statistic's low power in detecting heterogeneity, particularly when the number of studies is low. The $\mathrm{I}^{2}$ test statistic will be used to quantify statistical heterogeneity between studies, that is, provide a percentage of observed total variation across studies that is due to real heterogeneity rather than chance. This will provide a quantitative measure of heterogeneity. Cochrane provides the following rough guide to interpretation of heterogeneity: 0-40\%: might not be important; 30-60\%: may represent moderate heterogeneity; 50-90\%: may represent substantial heterogeneity; 75-100\%: considerable heterogeneity. ${ }^{33}$ If substantial heterogeneity is observed, we will try to explain the source of heterogeneity by subgroup analysis and/or sensitivity analysis.

\section{Subgroup analysis}

Subgroup analysis will be done in order to obtain estimates that are reflective, and thus potentially more useful and applicable, for specific subpopulation groups or settings, and will be conducted along the following strata, subject to availability of sufficient data: (1) schooling status-adolescents in school versus those not in school; (2) country of study participants; (3) age; (4) gender; (5) years of data collection, that is, 1990-1999, 2000-2016 and 1990-2016; and (6) diagnostic modality of LTBI and TB disease. The analysis along the strata of years of data collection, that is, 1990-1999 and 20002016, will be done in order to account for differences attributable to the advent of wide and free availability of antiretroviral therapy, although we appreciate that HIV prevalence is generally very low among adolescents as compared with adults.

\section{Sensitivity analyses}

Sensitivity analyses will be conducted to explore the source of heterogeneity, that is, determine the impact of specific studies on pooled prevalence estimate, by exclusion of studies with low-quality scores and thus higher risk of bias. We will also explore exclusion of studies with deficiency in specific items on the 10-point modified Hoy et al quality assessment tool, in order to evaluate the impact of this exclusion on pooled prevalence estimates.

\section{Ethics}

Given that we will use published anonymised data, which are publicly available and peer-reviewed, ethical approval is not required for this study.

\section{Dissemination (reporting of this review)}

Our review will be reported, as much as possible, in keeping with the PRISMA statement, ${ }^{34}$ and will include the PRISMA checklist (or adapted as practicable). Our findings will be published in a peer-reviewed journal and as part of a doctoral thesis at the University of Cape Town.

\section{Synthesis of evidence}

The PRISMA-P ${ }^{15}{ }^{16}$ recommends gauging of overall judgement of quality of evidence from obtained articles and indicates increasing support and use of the Grading of Recommendations Assessment, Development and Evaluation (GRADE) working group ${ }^{24}$ methodology. We will consider methodological quality of included studies and strength of evidence and adapt the basic principles of the GRADE approach.

\section{Author affiliations}

${ }^{1}$ The South African Tuberculosis Vaccine Initiative (SATVI), Division of Immunology, Department of Pathology, Institute of Infectious Disease and Molecular Medicine, University of Cape Town, Cape Town, South Africa ${ }^{2}$ School of Public Health and Family Medicine, University of Cape Town, Cape Town, South Africa

${ }^{3}$ Vaccines for Africa Initiatives, Institute of Infectious Diseases and Molecular Medicine, University of Cape Town, Cape Town, South Africa

${ }^{4}$ The Desmond Tutu HIV Centre, Institute of Infectious Diseases and Molecular Medicine, University of Cape Town, Cape Town, South Africa

\section{Twitter Follow Erick Bunyasi @ErickBunyasi}

Contributors EWB conceptualised and designed the study. EWB, B-MS, LHA, $\mathrm{HM}, \mathrm{MT}, \mathrm{AL}, \mathrm{JS}, \mathrm{TS}, \mathrm{HG}, \mathrm{RW}$ and $\mathrm{MH}$ were involved in the development of the study protocol. EWB prepared the first draft of the manuscript with supervision from HG, RW and MH. EWB, B-MS, LHA, HM, MT, AL, JS, TS, $H G, R W$ and $M H$ critically reviewed, revised and approved the subsequent and final version of the protocol. EWB is the guarantor. EWB and B-MS will perform the study search, screening and extraction of data under the guidance of $\mathrm{HG}$, RW and $\mathrm{MH}$.

Funding All authors have completed the ICMJE uniform disclosure at http:// www.icmje.org/coi_disclosure.pdf. The financial assistance of the National Research Foundation (NRF) towards the first author's PhD studies is hereby acknowledged. EWB initiated the research. This research is conducted as part of a PhD at the University of Cape Town. The first author (EWB) would also like to appreciate the supplementary departmental scholarship and support towards his PhD studies from the University of Cape Town (SATVI programme@UCT), of which this publication is a part.

Disclaimer Opinions expressed and conclusions arrived at are those of the authors and are not necessarily attributed to the NRF

Competing interests None declared.

Provenance and peer review Not commissioned; externally peer reviewed.

Data sharing statement The authors declare that this research protocol is an original work. Results from the study completed using this protocol will be published in a peer-reviewed journal.

Open Access This is an Open Access article distributed in accordance with the Creative Commons Attribution Non Commercial (CC BY-NC 4.0) license, which permits others to distribute, remix, adapt, build upon this work non- 
commercially, and license their derivative works on different terms, provided the original work is properly cited and the use is non-commercial. See: http:// creativecommons.org/licenses/by-nc/4.0/

\section{REFERENCES}

1. WHO U. The 2016 Global Tuberculosis Report. 2016. http://www. who.int/tb/publications/global report/en/ (accessed 28 Dec 2016).

2. Andrews JR, Hatherill $M$, Mahomed $\mathrm{H}$, et al. The dynamics of QuantiFERON-TB gold in-tube conversion and reversion in a cohort of South African adolescents. Am J Respir Crit Care Med 2015;191:584-91.

3. Mahomed $\mathrm{H}$. The incidence of tuberculosis in adolescents in the context of proposed TB vaccine trials. University of Cape Town, Theses 2013

4. Mahomed $\mathrm{H}$, Ehrlich $\mathrm{R}$, Hawkridge $\mathrm{T}$, et al. TB incidence in an adolescent cohort in South Africa. PLOS ONE 2013;8:e59652.

5. Mahomed H, Ehrlich R, Hawkridge T, et al. Screening for TB in high school adolescents in a high burden setting in South Africa. Tuberculosis (Edinb) 2013;93:357-62.

6. Andrews JR, Morrow C, Walensky RP, et al. Integrating social contact and environmental data in evaluating tuberculosis transmission in a South African township. J Infect Dis 2014;210:597-603.

7. Wood R, Lawn SD, Johnstone-Robertson S, et al. Tuberculosis control has failed in South Africa-time to reappraise strategy. SAMJ 2011;101:111-14.

8. Kwamanga D, Chakaya J, Sitienei J, et al. Tuberculosis transmission in Kenya: results of the third National Tuberculin Survey. Int J Tuberc Lung Dis 2010;14:695-700.

9. Wood R, Lawn SD, Caldwell J, et al. Burden of new and recurrent tuberculosis in a major South African city stratified by age and HIV-status. PLOS ONE 2011;6:e25098.

10. Middelkoop K, Bekker LG, Morrow C, et al. Decreasing household contribution to TB transmission with age: a retrospective geographic analysis of young people in a South African township. BMC Infect Dis 2014;14:221.

11. Mossong J, Hens $\mathrm{N}$, Jit $\mathrm{M}$, et al. Social contacts and mixing patterns relevant to the spread of infectious diseases. PLoS Med 2008;5:e74.

12. Wood R, Racow K, Bekker LG, et al. Indoor social networks in a South African township: potential contribution of location to tuberculosis transmission. PLOS ONE 2012;7:e39246.

13. Middelkoop K, Mathema B, Myer L, et al. Transmission of tuberculosis in a South African community with a high prevalence of HIV infection. J Infect Dis 2015;211:53-61.

14. Glynn JR, Guerra-Assunção JA, Houben RM, et al. Whole genome sequencing shows a low proportion of tuberculosis disease is attributable to known close contacts in Rural Malawi. PLOS ONE 2015;10:e0132840.
15. Moher D, Shamseer L, Clarke M, et al. Preferred reporting items for systematic review and meta-analysis protocols (PRISMA-P) 2015 statement. Syst Rev 2015;4:1.

16. Shamseer L, Moher D, Clarke M, et al. Preferred reporting items for systematic review and meta-analysis protocols (PRISMA-P) 2015: elaboration and explanation. BMJ 2015;349:g7647.

17. WHO U. Adolescent development. 2016. http://who.int/maternal child_adolescent/topics/adolescence/dev/en (accessed 6 Sep 2016).

18. RefWorks-COS P, ProQuest L. RefWorks. Ann Arbour, MI: ProQuest LLC, 2001.<QC: Please check with the PE as refs [18 and 29] are software references.>

19. Hoy D, Brooks $P$, Woolf $A$, et al. Assessing risk of bias in prevalence studies: modification of an existing tool and evidence of interrater agreement. J Clin Epidemiol 2012;65:934-9.

20. Werfalli M, Musekiwa A, Engel ME, et al. The prevalence of type 2 diabetes mellitus among older people in Africa: a systematic review study protocol. BMJ Open 2014;4:e004747.

21. Hussey HS, Abdullahi LH, Collins JE, et al. Varicella zoster virus-associated morbidity and mortality in Africa: a systematic review protocol. BMJ Open 2016;6:e010213.

22. Landis JR, Koch GG. The measurement of observer agreement for categorical data. Biometrics 1977:159-74.

23. StataCorp LP. STATA 14.1 SE. 2015. http://www.stata.com/ (accessed 18 Dec 2015)

24. GRADE. The Grading of Recommendations Assessment, Development and Evaluation (GRADE) working group. 2016. http:// www.gradeworkinggroup.org/ (accessed 11 Aug 2016).

25. Jüni $\mathrm{P}$, Witschi $\mathrm{A}$, Bloch $\mathrm{R}$, et al. The hazards of scoring the quality of clinical trials for meta-analysis. JAMA 1999;282:1054-60.

26. Higgins JP, Green S. Cochrane handbook for systematic reviews of interventions. Wiley Online Library, 2008.

27. Terracciano L, Brozek J, Compalati E, et al. GRADE system: new paradigm. Curr Opin Allergy Clin Immunol 2010;10:377-83.

28. Borenstein M, Hedges LV, Higgins J, et al. Front matter. Wiley Online Library, 2009.

29. Cochrane Collaboration. Review manager (RevMan) [computer program]. Copenhagen: The Nordic Cochrane Centre, The Cochrane Collaboration, 2011

30. Egger M, Davey Smith G, Schneider M, et al. Bias in meta-analysis detected by a simple, graphical test. BMJ 1997;315:629-34.

31. Higgins JP, Thompson SG, Deeks JJ, et al. Measuring inconsistency in meta-analyses. BMJ 2003:327:557-60.

32. Cochrane handbook. Heterogeneity in meta-analysis. 2016. http:// handbook.cochrane.org/chapter 9/9 52 identifying and measuring heterogeneity.htm (accessed 1 Jun 2016).

33. Ryan R, Hill S, Broclain D, et al. Cochrane consumers and communication review group. Study Design Guide, 2009.

34. Moher D, Liberati A, Tetzlaff J, et al. Preferred reporting items for systematic reviews and meta-analyses: the PRISMA statement. Ann Intern Med 2009;151:264-9. 